\title{
Uso de las Tecnologías de Información y Comunicación por parte de los Docentes de Instituciones Educativas de Sucre
}

\section{Use of Information and Communication Technologies by the Teachers of Sucre Educational Institutions}

\author{
DOI: http://dx.doi.org/10.179 81/econcuc.38.2.2017.10
}

Artículo de investigació. Recibido: ago 3/2017 Devuelto para revisión: sep 29/17 Aceptado: nov 06/2017

\author{
Marilú Tibisay Acurero Luzardo \\ María Elena Pérez Prieto \\ Alberto Luis Martínez Cárdenas ${ }^{3}$
}

\section{Resumen}

\begin{abstract}
El estudio esta centrado en el análisis del uso de las Tecnologías de la Información y la Comunicación-TIC en los docentes de instituciones educativas del municipio de Coveñas-Sucre. Es de tipo cuantitativa, descriptiva, no experimental y de campo. La población está conformada por once (11) instituciones educativas rurales y la muestra se tomó de manera intencionada, quedando representada por tres (3) instituciones. Los resultados evidencian una desviación estándar igual a uno (1.0), siendo un valor altamente confiable mostrando que los promedios calculados están cohesionados. Como conclusiones se llego a que el promedio (2.3) indica que el grado de conocimiento de los docentes en función de las TIC es relativamente bajo.
\end{abstract}

Palabras clave: Tecnologías de la Información y Comunicación, métodos educativos, procesos educativos, herramientas tecnológicas.

\begin{abstract}
The study focused on the analysis of the use of Information and Communication Technologies in teachers of educational institutions in the municipality of Coveñas-Sucre. It is quantitative, descriptive, not experimental, field. The population consists of eleven (11) rural educational institutions and the sample was taken intentionally, being represented by three (3) institutions. The results show a standard deviation equal to one (1.0), being a highly reliable value showing that the calculated averages are cohesive. As conclusions it found the average (2.3) indicates that the degree of knowledge of teachers in terms of ICT is relatively low.
\end{abstract}

Keywords: Information and Communication Technologies, educational methods, educational processes, technological tools.

\begin{abstract}
1 Posdoctora en Políticas Públicas y Paz Social. Doctora en Ciencias Gerenciales. Magister Sienciarum en Gerencia Financiera. Ingeniera de Sistemas. Docente Investigador del Grupo de Investigación "Estudios Socioeconómicos, Administrativos y ContablesESAC" de la Corporación Universitaria del Caribe-CECAR, Colombia. Categorizada por Colciencias nivel Asociado. Email: marilu.acurerol@cecar.edu.co; mariluacurero@hotmail.com
\end{abstract}

2 Doctora en Gerencia. Universidad Yacambu, Venezuela. Maestría en Gerencia de los Recursos Humanos. URBE, Venezuela. Licenciada en Administración, Mención Gerencia Industrial. UNERMB, Venezuela. Docente Investigador del Grupo de Investigación "ESAC" de la Corporación Universitaria del Caribe-CECAR, Colombia. Email: maria.perezpr@cecar.edu.co

3 Especialista en Gerencia del Talento Humano. Miembro de la línea de investigación Sistemas de información en el campo de las Ciencias Sociales. Docente Investigador del Grupo de Investigación "ESAC" de la Corporación Universitaria del CaribeCECAR, Colombia. Email: alberto.cardenas@cecar.edu.co 


\section{Introducción}

La tecnología, constituye una clave para la excelencia académica, es mediante el uso de herramientas informáticas de comunicación como el docente puede reunir, almacenar, transmitir, procesar y recuperar electrónicamente caracteres numéricos, así como alfanuméricos, imágenes, videos, sonido, y medios electrónicos, para el control de sus actividades investigativas e interpretar la realidad a partir de elementos concretos de información. La globalización de la economía, la sociedad de la información, la aceleración de cambios tecnológicos y las nuevas formas de trabajo, exige tanto a los docentes como a las instituciones de educación superior ampliar su capacidad para el manejo de esta información y una mayor eficiencia tecnológica.

En este sentido Martínez y Solano (2011), considera a la Tecnología de la Información y Comunicación-TIC como la estrategia para llevar adelante el desarrollo de la globalización; es mediante el uso de las redes de comunicación y servicios de telecomunicaciones que la interconexión social ha sido posible, creando nuevos espacios de acción y diversos fenómenos sociales que la educación como proceso de transformación humana debe abordar. Esto ha generado en los distintos organismos internacionales, nacionales y regionales, vinculados con el desarrollo y progreso de las naciones, que orienten esfuerzos para el fomento del proceso de institucionalización del sistema universitario formal.

Por otra parte, el Centro Interamericano para la Planificación de la Educación en América Latina-CEPAL (2016), institución en el cual se revisa la evolución de los mercados de trabajo de la región se espera que para el primer semestre de 2016 sea poco favorable las perspectivas de crecimiento regional, apenas por el orden del $1.1 \%$, lo cual refleja una persistente debilidad de la demanda laboral y la generación de empleo asalariado. El mismo organismo expresa que solamente con políticas de desarrollo productivo claras en la región, se podrá superar el contexto adverso que obstaculiza la expansión y generar más y mejores empleos para la inserción productiva de la fuerza laboral (CEPAL, 2016).

La nueva dinámica de la sociedad demanda individuos no sólo con pensamiento crítico y formación ética para contribuir con el desarrollo sostenible, sino también con capacidades sólidas para sobrevivir en un mercado laboral cada vez más exigente. Por esta razón, el rol de las instituciones y su pertinencia es actualmente un tema de discusión entre los académicos. La brecha entre el mercado de trabajo y la educación genera polémica entre los expertos y sobre cómo solucionarlo (UNESCO, 2015).

Al observar este panorama y teniendo en cuenta que la educación de los jóvenes hoy día se enfrenta a desafíos y dificultades relativos a una mejor capacitación del docente, cuestión que se refleja en la formación de los estudiantes sobre todo en sus competencias, Es necesario mejorar y conservar la calidad de la enseñanza, la investigación, los servicios y la pertinencia de los planes de estudios, el establecimiento de acuerdos de cooperación, entre otros aspectos; permitiendo hacer frente a los retos del mundo global, aprovechando además, nuevas oportunidades que abren las TIC al mejorar la manera de producir, organizar, difundir, acceder y controlar el conocimiento para incidir de manera directa en la integración del campo laboral.

Por consiguiente, la educación para hacerle frente a los retos del mundo global y del desarrollo tecnológico, debe comenzar un proceso de transformación y renovación, contribuyendo al desarrollo de la región. Debe iniciarse con la actualización del docente, el aprendizaje permanente de nuevos conocimientos, la promoción y generación de la investigación científica y tecnológica en las 
aulas, con el propósito de poder transmitir de manera eficiente el conocimiento apropiado a los alumnos que se enfrentan a un mundo laboral (Tolentino, 2014).

Las consideraciones expresadas inducen a la búsqueda de alternativas y diferentes medios tecnológicos que puedan ser utilizados como herramientas en el que hacer docente; contribuyendo a lograr una enseñanza de calidad, donde mantenga actualizado el conocimiento que los estudiantes necesitan aprender. Para tal fin, sus miembros deben sentirse motivados, satisfechos y comprometidos con la institución; conscientes de un proceso de auto evaluación constante dependiente del entorno, con la finalidad de lograr una mayor productividad, eficiencia, eficacia y mejor servicio en la institución, que se traduce en una educación con valores, donde los docentes se consideran inmersos en esta sociedad del conocimiento.

En tal sentido, las instituciones educativas, deben aportar a la sociedad recursos humanos, capaces de usar y aplicar las nuevas tecnologías en los diversos campos de la actividad humana; reconocer los impactos $\mathrm{y}$ transformaciones que ocasionan, así como ver la manera de aprovechar la tecnología de la información, para lograr un aprendizaje continuo. Ante el fenómeno, sociedad de la información, el sector educativo tiene una importancia crítica y fundamental, debido al proceso formativo de los recursos humanos que se efectúa en este contexto. Por esta razón, los gestores de las instituciones educativas deben ser capaces de producir cambios y promover una nueva forma de concienciadirigida a orientar y ejecutar las políticas de desarrollo nacional y regional de cada país, acorde con las nuevas exigencias mundiales.

Para lograrlo, se plantea la necesidad del uso de las TIC, consideradas como el conjunto de procesos y productos derivados de muchas herramientas constituidas en soportes de la información y canales de comunicación, relacionados con el almacenamiento, procesamiento y trasmisión digitalizada de la información. Razón por la cual, las TIC se convierten en verdaderos elementos facilitadores para todas aquellas personas e instituciones interesadas en superar las sucesivas interrogantes presentadas en el acto comunicativo del proceso de enseñanza y aprendizaje creciente actualmente con mucha rapidez.

No obstante, los docentes de las instituciones educativas rurales de Sincelejo (SucreColombia) mostraron debilidades en cuanto al manejo de herramientas tecnológicas que se reflejan en la poca interacción con las TIC. Los estudiantes manifestaron que el uso de las computadoras en el aula de clases solo se hacía de manera eventual en ocasiones en que el docente responsable de informática utilizaba alguna de las computadoras encontradas bajo resguardo para explicar la clase correspondiente. Esto refleja una problemática de la gestión académica, la cual no responde eficaz y oportunamente a los cambios generados por las tecnologías de la información y comunicación emergentes. Tal como se evidencia, existe poca comunicación por Internet y pocos equipos de computación operativos en las instituciones educativas rurales (Acurero, Rivera, Hernández y Cáceres, 2017).

Según lo expuesto, el personal docente está dispuesto a adquirir nuevos conocimientos, en función del desarrollo tecnológico del momento, de allí la necesidad de que las instituciones deban caracterizarse por la manera en como generan y transforman el conocimiento, a través del uso de la tecnología de información y comunicación del momento. Para lograr la calidad, el docente debe desarrollar habilidades, formar actitudes, fomentar mecanismos de aprendizaje en un espacio de acción tecnológica, reflejando la necesidad de elaborar estrategias apropiadas de planificación y análisis, asegurando una enseñanza, formación e investigación de gran calidad, logrando con ello la integración con la sociedad. 
El manejo de la información, se considera como una herramienta para gestionar con anticipación y encaminar el conocimiento según los cambios fundamentales en la forma de accederla, obtenerla y administrarla. El desarrollo informático, la utilización de computadores y el trabajo en redes de comunicación, permiten intercambiar y difundir la información, dando lugar a una comunicación interactiva. Asimismo, con una efectiva comunicación se desarrollan nuevas formas de trabajo, individuales o en equipo, donde existe un continuo enriquecimiento y adaptación permanente de la información que emerge de las nuevas tecnologías, dentro de los parámetros establecidos de organizar y mantener un trabajo eficaz.

\section{Las Tecnologías de la Información y la Comunicación}

Las Tecnologías de Información y Comunicación - TIC son consideradas como un conjunto de herramientas y recursos tecnológicos utilizados para crear, diseminar, almacenar, y gestionar información, a fin de comunicarla; inciden directamente en la educación, dado que se ha venido observando en los últimos años, que el acceso a herramientas tecnológicas de información y comunicación digital, a aplicaciones, redes y multimedia ha crecido de manera exponencial (UNESCO, 2015).

A medida que las instituciones, empleen las TIC para resolver problemas competitivos y administrar los trabajos internos de la organización, se creará un nuevo conjunto de normas organizacionales. En un momento dado, surgirá una nueva cultura en la que serán fundamentales los diversos aspectos de las tecnologías y las personas que los dominan. La automatización de los procesos, la elaboración de sistemas automatizados se pone de manifiesto y en muchas áreas el personal encontrado en el nivel técnico tiene mayor conocimiento del uso de las herramientas tecnológicas (Acurero, 2016).
Para llegar al alcance de esta política el Ministerio de Educación Nacional (2012) ha diseñado programas que promueven estrategias para la formación de docentes mediante una ruta, con la cual se va desde la apropiación personal de las TIC para hacer un uso básico de ellas, hasta la apropiación profesional para su uso pedagógico, beneficiándose docentes de la educación básica, media y del ciclo complementario de las Escuelas Normales. Es de anotar que dichas políticas no tienen alcance en el contexto universitario, por lo cual se delega en estas instituciones la responsabilidad de la formación de sus docentes en el campo de las TIC y su uso pedagógico.

Por tanto es evidente que las TIC constituyen un pilar fundamental de la educación y representan un nuevo escenario para comprender el fenómeno educativo, que implica una nueva cultura educativa y pedagógica. Ofreciendo un apoyo valioso para este propósito ya que su uso fortalece procesos de interacción e intercambio de información en entornos apropiados y en diversos contextos que ayudan a la movilización de saberes, rompiendo con el aula tradicional y favoreciendo la cooperación entre los diferentes actores.

No obstante, las TIC tienen la capacidad apropiada del procesamiento y almacenamiento de la información, ofreciendo programas funcionales de operación, así como conexiones a redes, fijas y móviles, con la integración de aplicaciones que posibilitan conectar programas, permitiendo al usuario transferir y compartir información entre ellos, facilitando la transferencia de conocimiento entre distintos equipos y plataformas interconectadas y trabajando de forma coordinada. En este entendido, la incorporación de las TIC en las instituciones educativas requieren de su uso y apropiación por parte de los estudiantes y docentes, e igualmente es necesario que el profesor ajuste sus actividades utilizándolas como medio para construir conocimientos específicos en un área determinada (Chumaceiro, Ramos y Hernández, 2017). 
Dentro del ámbito de la alfabetización digital se presenta una gran diversidad de definiciones sobre esta, que van desde las más simples y elementales a otras más integrales, complejas y comprensivas. Gross y Contreras (2006) la consideran "como el conjunto de habilidades socio-cognitivas mediante las cuales se puede seleccionar, procesar, analizar e informar del proceso de transformación de información a conocimiento" (2006).

Por su parte, Caicedo, Montes y Ochoa-Angrino (2013) complementan esta idea al afirmar que implica crear en los individuos competencias para reconocer sus necesidades de información y satisfacerlas por medio de la localización, evaluación y el uso eficiente de la información, así como la creación de habilidades que favorezcan el autoaprendizaje durante toda la vida.

De igual manera, según lo contemplado por la UNESCO (2015) se deben generar las competencias en los estudiantes para que sean capaces de buscar, analizar y evaluar información, solucionar problemas, tomar decisiones, utilizar la creatividad, comunicar, colaborar, publica, producir, contribuir a la sociedad, siendo ciudadanos y ciudadanas informados y responsables. Pudiéndose apreciar claramente como en este mundo del movimiento, del cambio y de la transformación constante, es necesario un conocimiento abierto, competente y competitivo, capaz de modificarse a sí mismo, siendo la educación el pasaporte de los individuos y de las naciones a la sociedad del conocimiento y a la aldea global

Estas tecnologías, son cada vez más accesibles y juegan un papel fundamental en todo el mundo. El acceso a películas, videos, teléfono, televisión o radio, es mucho más frecuente que el acceso a un computador, internet y a la World Wide Web. Las nuevas TIC, no son tecnologías únicas, sino combinaciones de hardware, software, soportes, y sistemas de distribución (Palacios, 2000), caracterizadas por una integración de múltiples soportes, interactividad, flexibilidad de utilización y conectividad.

$\mathrm{Al}$ poder integrarse en múltiples soportes con una sola aplicación educativa, se produce la interactividad e incluyen la posibilidad de controlar, manipular y contribuir al contenido informativo y al ser flexibles ofrecen libertad frente a los horarios rígidos y las limitaciones de tiempo y espacio, y por medio de la interconexión cualquier persona a que tenga conexión a internet, desde cualquier lugar del mundo puede acceder a cientos o miles de archivos de información y a millones de páginas Web (Blurton, 2000).

Así pues, se considera a las nuevas Tecnologías de Información y Comunicación, como los medios colectivos para agrupar almacenar, transmitir, procesar y recuperar electrónicamente palabras, números, imágenes y sonidos, así como a los medios electrónicos para controlar equipos de toda especie. La implantación de estas tecnologías siempre reclaman cambios en la organización humana; como mínimo se hace necesaria la capacitación del personal directivo y docente. Una de sus aportaciones potenciales es la capacidad de respaldar modos de trabajo que no serían nada prácticos con métodos manuales de trabajo o sistemas automatizados de generaciones anteriores. Los cambios de esta naturaleza requieren mucho más que una simple capacitación del personal para operar el nuevo equipo. Existen ejemplos de modos de trabajo diferentes con implicaciones que van mucho más allá del grupo inmediato de trabajo.

De lo expuesto, se evidencia que las TIC, incluyen herramientas como equipos electrónicos, computadoras, teléfono, videos, cámaras digitales y copiadoras, entre otros. Además, su uso puede extenderse al terreno de las imágenes, como la arquitectura, ingeniería, gráficas, modas y películas; también, se incluye la música e innumerables aplicaciones, las cuales al ser utilizadas inducen a una serie de cambios. 
A medida que las instituciones empleen las TIC para resolver sus problemas, se hacen más competitivas y pueden administrar mejor los trabajos internos de la organización, se crean nuevos conjunto de normas organizacionales, se benefician los procesos de trabajo mejorando la infraestructura de la organización, se vuelven más efectivos y eficientes en el intercambio de la comunicación y la información; haciendo que en un momento dado, surja una nueva cultura en la que seran fundamentados los diversos aspectos de la tecnología de la información y las personas que los dominan. La automatización de los procesos, la elaboración de sistemas computarizados de información y el uso de las tecnologías en general se ponen de manifiesto en estas organizaciones.

\section{Las Tecnologías de Información \\ y Comunicación y los docentes \\ de las instituciones educativas}

La educación asistida por las TIC considera la formación académica impartida mediante el uso permanente de herramientas tecnológicas, que incluyen el manejo de un canal tecnológico de comunicación, como la televisión, la radio, o una red de computadoras. También existen aplicaciones y métodos que favorecen al aprendizaje, donde se anima a los estudiantes a trabajar con entornos ricos en información y experiencias para llegar a la comprensión de los procesos formativos por sí mismos.

Para poder utilizar las tecnologías en las instituciones deben equiparse con computadoras conectadas en redes que permitan el acceso a internet, tomando en consideración además las redes de comunicación que tienen componentes educativos, los cuales beneficien a las instituciones. Países desarrollados cuentan con instituciones educativas que poseen centros de computación con acceso a internet para uso de los estudiantes y profesores en la clase; permaneciendo abierto fuera del horario de la escuela y los alumnos y profesores forman a los padres y otros miembros de la comunidad que no tengan acceso a internet. Así, la escuela facilita el acceso a internet a la comunidad, con algún beneficio obtenido por la prestación del servicio (Blurton, 2000).

La utilización dada a los computadores en la educación depende de la capacidad pedagógica y de los conocimientos técnicos de los profesores, que deben saber cómo explotar estas modernas tecnologías de forma que tengan un significado educativo. En algunos países, como por ejemplo el Reino Unido, para lograr la titulación de profesor es necesaria la formación en el uso de las TIC. Esto, junto con la medida en que evolucionan las aplicaciones educativas de las TIC, además de la formación preparatoria, se hacen necesarios cursos de reciclado para profesores con experiencia.

La formación de profesores en las TIC debe contemplar, según la UNESCO (2015), al menos dos aspectos: En primer lugar, los profesores necesitan formación técnica para aprender a utilizar y mantener los equipos y software; esta formación se está dando de muy distintas formas con cursos previos a la ocupación del puesto, seminarios en el puesto de trabajo, propagandas comerciales de formación, así como otras formas, muchas de las cuales utilizan las TIC para dar la formación. En segundo lugar, puesto que la integración de las tecnologías en los programas educativos exige grandes cambios, es necesario dar formación sobre cómo integrarlas en los programas, los cuales deben incluir métodos eficaces de enseñanza basada en las TIC y la utilización de aplicaciones específicas para asignaturas concretas.

Ahora bien, Schalk (2011) en el informe de la UNESCO-IESALC en un intento por establecer un posible esquema para la formación de profesores, identifica las áreas relacionadas con el conocimiento de aplicaciones concretas; tales como la integración en los programas existentes, en los cambios de los programas en relación con las TIC, y los cambios en el papel del profesor y teorías pedagógicas de 
apoyo. Evidenciando que en la mayoría de los casos la mayor parte de la formación de los profesores se centra exclusivamente en el los primeros aspectos.

También es necesario señalar que actualmente se están desarrollando muchas formas innovadoras para dar apoyo al personal docente, donde se han establecido programas de formación tecnológicas, permitiendo combinar la informática con la enseñanza. Igualmente se hace necesaria la contratación de personal cualificado en una disciplina académica y con experiencia en la utilización de las TIC en educación, para proporcionar al profesorado asesoría y apoyo en información y tecnología con fines educativos para estimular su aprendizaje y concientización en cuanto al uso de la tecnología, tanto en la institución como fuera de ella.

Las metodologías de formación varían, pero los modelos de formación de formadores son comunes y en la mayor parte de los casos resultan más rentables que la formación in situ individual o a pequeños grupos. En estos casos se seleccionan "profesores-líderes" siguiendo una serie de criterios, como experiencia previa con las TIC en educación, experiencia en desarrollo de personal y compromiso con el programa por parte de las autoridades escolares y locales. Estas personas reciben cursos intensivos para perfeccionar los mecanismos y formas de integración de las TIC en los programas.

Una vez formados, vuelven a sus centros y dan formación y apoyo a sus colegas, obteniendo además una formación continuada y de apoyo de larga duración, y se realiza una vinculación directa mediante conferencias o capacitaciones virtuales que continuamente permite un seguimiento personalizado de cada docente. Esta manera de interactuar, es conveniente y fundamental en zonas geográficas distantes y con una gran extensión, la responsabilidad de esta formación y apoyo continuado recae en los mismos docentes y directivos de las instituciones.
En esta medida se refleja a las TIC como inmersas en la sociedad de la información y el conocimiento, consideradas promotoras del cambio social, contribuyendo a eliminar fronteras y barreras, a difundir la cultura, a potenciar la participación social, permitiendo la creación de espacios de oportunidades (Pérez y Sarrate, 2011). El uso de las TIC supone un elemento clave en el desarrollo de las personas donde no hay exclusión. Por tanto, facilitar el acceso a estas nuevas herramientas a los colectivos más desfavorecidos es decisivo para su integración, su participación e inserción social dentro del ámbito de las comunidades y el campo laboral.

El desarrollo profesional de los estudiantes, depende de la preparación de los docentes y la calidad de la educación, provenientes de las transformaciones de las prácticas educativas con el apoyo de las TIC, a fin de adoptar estrategias orientadas a los estudiantes hacia el uso de las TIC que generen cambios positivos sobre su entorno, y promuevan la transformación de las instituciones educativas en organizaciones de aprendizaje. Esto conlleva a la transformación de la sociedad (el trabajo, el mercado, los procesos productivos, la política, la educación, la vida cotidiana...) de tal manera que cuando existan individuos, países o regiones que tienen acceso a ellas, se genera un espacio de interconexión disminuyendo la brecha digital existente.

El desarrollo de la tecnología, la apertura de los mercados a través de los procesos de globalización, las crisis sociales, políticas y económicas dentro de todas las sociedades, establecen la necesidad de repensar los procesos gerenciales para enfrentar las nuevas tendencias caracterizantes del futuro inmediato en donde operan las organizaciones.

Dentro de este panorama de gran compromiso, los directivos de las instituciones deben propiciar la creación de un ambiente de trabajo que como criterio principal realice evaluaciones continuas de sus acciones y las articule coherentemente, en función de lograr 
la armonía deseada en cuanto al desarrollo tecnológico requerido, garantizando la calidad característica de los procesos académicos, a fin de lograr de esta forma un alto desarrollo y productividad internos. De igual manera se observa como los docentes son motivados para el uso de las tecnologías, propias de un mundo globalizado, de tal manera que los estudiantes en un tiempo temprano puedan insertarse al desarrollo de actividades conjuntas propuestas por los docentes evidenciando avances significativos en los aprendizajes adquiridos (Cool y Martí, 2001).

Sobre la base de lo expuesto, es imperante que los jóvenes en formación, sobre todo aquellos que habitan en zonas rurales y de remoto acceso tengan la posibilidad de interactuar y comprender el uso de las herramientas que la sociedad moderna exige para su funcionamiento, pues esto condiciona su incursión en el mercado laboral, que es un ambiente cada vez más cambiante y complejo, donde las organizaciones exigen habilidades especificas en el uso de tecnologías funcionales para el desempeño en los puestos de trabajo. Todo esto es posible solo si el docente que es llamado a formarlo tiene el nivel de conocimiento adecuado de estas TIC y los familiariza con su uso y aprovechamiento.

\section{Metodología}

La investigación es de tipo cuantitativa, descriptiva, no experimental y de campo. La población del estudio está conformada por once (11) instituciones educativas rurales del $\mathrm{Mu}$ nicipio Coveñas-Sucre y la muestra se calculó según la fórmula estadística descriptiva, quedando representada por tres (3) instituciones, las cuales fueron seleccionadas de manera intencional tomando en consideración el número de estudiantes y el número de profesores.

En las instituciones seleccionadas se procedió a aplicar el instrumento para la recolección de la información, el cual previamente había sido aplicado por Acurero (2016) cumpliendo con las validaciones respectivas con un coeficiente de Alpha Cronbach de 0.956. El instrumento se aplicó a todos los docentes de las instituciones educativas, y contiene una serie de preguntas con una escala tipo Licker basada en cinco alternativas, aplicado a los docentes de las (30) diferentes instituciones educativas.

El cuestionario empleado da respuesta a los indicadores del estudio referidos a las herramientas de comunicación, los métodos educativos, los programas educativos y la infraestructura tecnológica. El procesamiento de los datos se realizó a través del cálculo de promedios y desviaciones estándar, que permitieron realizar el análisis pertinente en cuanto al estudio realizado.

\section{Resultados}

Para dar respuesta al objetivo del estudio enfocado a analizar el uso de las TIC por parte de los docentes de las instituciones educativas de Coveñas (Sucre, Colombia), se presentó para cada indicador los resultados obtenidos según los promedios arrojados de acuerdo al procesamiento estadístico de datos. En cuanto a las herramientas de comunicación se uso el teléfono, el computador y el internet (propio o de la institución). Para los métodos educativos se verificó que los docentes utilizan el computador como herramienta de estudio, así como los softwares educativos. Igualmente se consultó si se estimula a los estudiantes a comunicarse a través de medios electrónicos y sobre el uso de las bases de datos virtuales.

Para la infraestructura tecnológica se verifica que la institución cuenta con equipos de computo y redes de comunicación propios, para que los docentes realicen difusión de sus programas educativos a distancia; también se considera la apropiación del conocimiento por parte de los docentes como desarrollo profesoral referidos a métodos y programas educativos virtuales; así como la existencias de convenios de cooperación con comunidades virtuales o la participación en redes nacionales e internacionales de vinculación. 


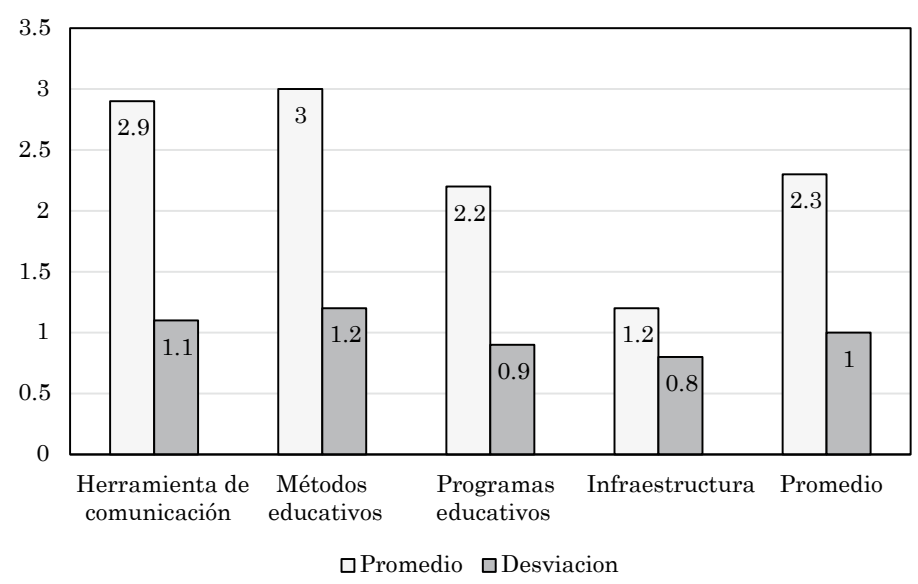

Figura 1. Uso de las Tecnologías de Información y Comunicación. Fuente: Autore.

Los resultados de los promedios obtenidos en el análisis de la información evidencian una desviación estándar igual a uno (1.0), reflejando un valor altamente confiable significando que los resultados obtenidos en función de los promedios calculados están cohesionados. El promedio general (2.3) según la opinión de los encuestados, indica que el grado de conocimiento de los docentes en función del uso de las TIC, no es el más apropiado.

No obstante, en lo respectivo a los indicadores herramientas de comunicación (2.9) y métodos educativos (3.0), ambos valores están por encima del promedio general indicando que las TIC son usadas moderadamente; no así los programas educativos y la infraestructura tecnológica con (2.2) y (1.2) respectivamente, reflejando muy poca frecuencia de uso, pudiéndose contactar que la institución no cuenta con una infraestructura apropiada de redes de comunicación y de internet. $\mathrm{Al}$ respecto la formación de profesores en las TIC la UNESCO (2015), manifiesta que los docentes necesitan formación técnica para aprender a utilizar y mantener los equipos y softwares educativos; así como integrar los programas educativos y los métodos eficaces de enseñanza basadas en las TIC y la utilización de aplicaciones específicas para asignaturas concretas.
Así mismo, para Tolentino (2014) la educación debe comenzar un proceso de transformación y renovación tecnológica; contribuir al desarrollo de la región, iniciando con la actualización docente, que invita al aprendizaje permanente de nuevos conocimientos, incentivándolos a la investigación científica y tecnológica en las aulas, con el propósito de poder transmitir de manera eficiente el conocimiento apropiado a los alumnos

\section{Conclusiones}

El estudio determinó la necesidad de perfeccionar los conocimientos adquiridos de los docentes de las instituciones educativas en cuanto al uso de las TIC, clasificadas según los indicadores como herramientas de comunicación, métodos educativos, programas educativos y la infraestructura tecnológica. Los resultados reflejan en términos generales un uso poco frecuente de las TIC por parte de los docentes de las instituciones educativas estudiadas.

Es necesario lograr la integración estratégica y tecnológica de cada una de las instituciones educativas, orientadas hacia una mejora continua de los métodos y procesos educativos, la flexibilización del currículo, herramientas tecnológicas y la dotación de equipos de computación y redes de comunicación. 
Se debe además responder a los continuos cambios de la sociedad de la información, la cual demanda de docentes con una amplia capacitación tecnológica que sean capaces de generar, promover y difundir el conocimiento.

El compromiso está en los rectores de las instituciones educativas que deben estar a la vanguardia del uso de las Tecnologías de Información y Comunicación, con el propósito de combinar las diferentes herramientas tecnológicas con los recursos humanos y técnicos, con una visión gerencial de los procesos involucrados en el que hacer educativo.

\section{Referencias}

Acurero, M. (2016). Gestión Académica en las Universidades a través de las Tecnologías de Información y Comunicación. Cabimas: Fondo Editorial UNERMB.

Acurero, M., Rivera, T., Cáceres, A. y Hernández, J. (2017). Competencias emprendedoras desarrolladas a partir de herramientas tecnológicas aplicadas en los jóvenes de las instituciones educativas rurales del Municipio Sincelejo - Sucre. En, CIMTED, Las competencias y la gestión del conocimiento. (294-307). Medellín: Corporación CIMTED.

Blurton, C. (2000). Nuevas Tendencias en Educación. Informe Mundial sobre la Comunicación y la Información (1999 - 2000). España: Ediciones UNESCO / CINDOC.

Caicedo, A., Montes, J. y Ochoa-Angrino, S. (2013). Aprender de y con la tecnologia: algunos resultados de investigacion sobre la integracion de las TIC en la educacion superior. Carta de AUSJAL, 38, 28-35.

Cool, C. y Martí, E. (2001). La educación escolar ante las nuevas tecnologías de la información y la comunicación. Desarrollo psicológico y educación. Psicología de la educación escolar. Madrid: Alianza.
CEPAL. (2016). Perspectivas económicas de América Latina 2016. Recuperado de https://repositorio.cepal.org/bitstream/ handle/11362/39535/S1501061_es.pdf

Chumaceiro, A., Ramos, F. y Hernández, J. (2017) Los Procesos Formativos para el Siglo XXI. Cabimas: UNERMB.

Gross, B. y Contreras, D. (2006). La alfabetización digital y el desarrollo de competencias ciudadanas. Revista Iberoamericana de Educación, (42), 103-125.

Martínez, F. y Solano, I. M. (Coord)(2011). Comunicación y Relaciones Sociales de los Jóvenes en la red. Alcoy: Marfil.

Palacios, G. (2000). Implicaciones de las Nuevas Tecnologías de la Información y la Comunicación en la Educación. Monterrey: ITESM.

Pérez, M. y Sarrate, M. (2011). Las TIC promotoras de inclusión social Revista Española de Pedagogía, 69, (249), 237-254.

República de Colombia. Ministerio de Educación. (2012). Políticas de integración de TIC en los sistemas educativos. Recuperado de https://www.mineducacion. gov.co/cvn/1665/articles-311722_archivo9_pdf.pdf

Schalk, A. (2011). La Universidad y el Campo Laboral. [Boletin Iesalc de Educación Superior. No. 216]. UNESCO-IESALC.

Tolentino, L. (2014). Desempeño Didáctico y Académico del Docente Relacionado a la Satisfacción de los Estudiantes del Programa de Complementación Pedagógica de la Universidad Nacional Mayor de San Marcos, 2013 - II. [Tesis]. Universidad Nacional Mayor de San Marcos, Lima, Perú.

UNESCO. (2015). El impacto de las TIC en la educación superior. Relatoría de la conferencia nacional de Brasilia. Recuperado de http://unesdoc.unesco.org/ images/0019/001905/190555s.pdf 